\title{
Espécies de Chalcididae (Hymenoptera, Insecta) do Estado do Espírito Santo, Brasil
}

\author{
Marcelo Teixeira Tavares \& Bruno Cancian de Araujo
}

Biota Neotropica $v 7$ (n2) - http://www.biotaneotropica.org.br/v7n2/pt/abstract?inventory+bn02207022007

\author{
Recebido em 01/09/06 \\ Versão Reformulada recebida em 15/03/07 \\ Publicado em 04/06/07 \\ Departamento de Ciências Biológicas, Universidade Federal do Espírito Santo - UFES, \\ Av. Marechal Campos, 1468, CEP 29.040-090, Vitória, ES, Brasil \\ Autor para correspondência: Marcelo Teixeira Tavares,e-mail: mtavares@npd.ufes.br; \\ Bruno Cancian de Araujo, e-mail: cancianrally@gmail.com
}

\begin{abstract}
Tavares, M.T. \& Araújo, B.C. Chalcididae (Hymenoptera, Insecta) species from Espírito Santo State, Brazil. Biota Neotrop. May/Aug 2007 vol. 7, no. 2. http://www.biotaneotropica.org.br/v7n2/pt/abstract?invento ry+bn02207022007. ISSN 1676-0603.

A checklist of Chalcididae (Hymenoptera, Insecta) species is presented for the state of Espírito Santo, Brazil. Specimens were collected in 22 localities and belong to 37 species and 11 genera. Only two species and five genera have been previously recorded for the state. Seven species and one genus are recorded for the first time for Brazil and two species are new records for the Neotropical region. The geographic distribution of these species is discussed.

Keywords: checklist, parasitic wasps, geographic distribution, Neotropical.

Resumo

Tavares, M.T. \& Araújo, B.C. Espécies de Chalcididae (Hymenoptera, Insecta) do Estado do Espírito Santo, Brasil. Biota Neotrop. May/Aug 2007 vol. 7, no. 2. http://www.biotaneotropica.org.br/v7n2/pt/abstract? inventory+bn02207022007. ISSN 1676-0603.

Uma lista das espécies de Chalcididae (Hymenoptera, Insecta) é apresentada para o Estado do Espírito Santo. Os exemplares são procedentes de 22 localidades e pertencem a 37 espécies e 11 gêneros, sendo que somente duas espécies e cinco gêneros já haviam sido registrados para o Estado. Sete espécies e um gênero são novos registros para o Brasil e duas espécies são registradas pela primeira vez para a região Neotropical. A composição desta fauna é discutida em relação à distribuição geográfica das espécies.
\end{abstract}

Palavras-chave: lista de espécies, vespas parasitóides, distribuição geográfica, Neotropical. 


\section{Introdução}

As vespas da família Chalcididae são himenópteros parasitóides bastante peculiares. O corpo apresenta cor e tamanho variáveis. Muitas espécies neotropicais apresentam o corpo amarelo (Figura 1), vermelho ou castanho, freqüentemente manchado de negro. Outras têm o corpo castanho, marrom-avermelhado ou negro e, eventualmente, as formas negras podem apresentar manchas claras ou reflexos metálicos. O tamanho do corpo varia de cerca de 1,5 $\mathrm{mm}$ de comprimento (e.x. Notaspidium) até cerca de 23,0 mm (e.x. Acanthochalcis), estando entre os maiores calcidóideos conhecidos. As pernas posteriores são mais desenvolvidas que as outras, as coxas são espessas e os fêmures dilatados com uma fileira de dentes ou um pente de dentículos ventrais, que se acopla à tíbia recurvada. Estas pernas são utilizadas para sustentar o corpo da fêmea, segurar ou se defender do hospedeiro durante a oviposição e, eventualmente, agredir fêmeas concorrentes durante a disputa por hospedeiros (Cowan 1979).

Suas espécies atuam como parasitóides de diversas ordens de holometábolos. A maioria é parasitóide primário de Lepidoptera, Diptera, Coleoptera ou Hymenoptera, e atacam seus hospedeiros no estágio de larva madura ou de pupa. Algumas são hiperparasitóides obrigatórios de Ichneumonoidea (Hymenoptera) e Tachinidae (Diptera). Bouček (1988) menciona a ocorrência de parasitismo em Strepsiptera de vida livre. Biologia peculiar é a de espécies de Chalcis que se desenvolvem como parasitóides primários, solitários e cenobiontes de Stratiomyidae (Diptera), atacando o hospedeiro na fase de ovo, ou larva jovem, e emergindo na fase de pupa (Cowan 1979).

Perioto \& Tavares (1999) mencionam que, apesar da maioria de seus gêneros estar restrita ao Velho Mundo, esta família apresenta maior riqueza de espécies no Novo Mundo. A família conta com 86 gêneros e 1743 espécies nominais distribuídas ao redor do mundo (Arias \& Delvare 2003).

O Estado do Espírito Santo apresenta áreas com alto índice de riqueza já detectado para vários grupos biológicos (Thomaz \& Monteiro 1997, Brown Jr \& Freitas 2000, Mendes \& Padovan 2000, Azevedo \& Santos 2000, Passamani et al. 2000), porém, apenas cinco gêneros (Azevedo et al. 2002) e duas espécies (Halstead 1991) de Chalcididae foram registrados para o Estado até o momento.

Neste estudo novas ocorrências de gêneros e espécies de Chalcididae são registradas para a fauna do Estado do Espírito Santo e a composição da fauna é comparada com a de outras regiões.

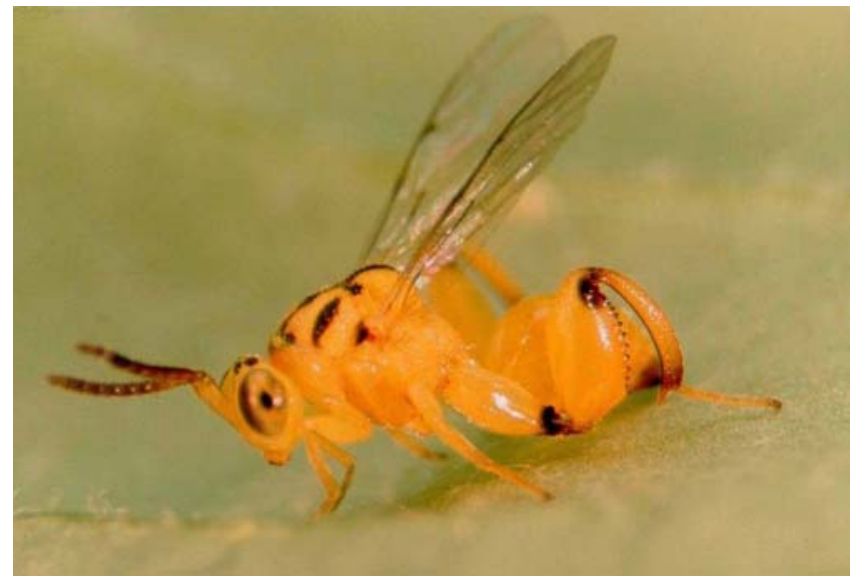

Figura 1. Adulto de Conura sp.

Figure 1. Conura sp, adult.

\section{Material e Métodos}

Foram estudados 507 exemplares procedentes de 22 localidades do Espírito Santo e que estão depositados nas seguintes coleções: CASC, California Academy of Sciences, São Francisco, EUA; IBGE, Coleção Zoológica da Reserva Ecológica do Instituto Brasileiro de Geografia e Estatística, Brasília, Brasil; IBSP, Instituto Biológico, São Paulo, Brasil; INPA, Instituto Nacional de Pesquisas da Amazônia, Manaus, Brasil; MBML, Museu de Biologia Professor Mello Leitão, Santa Teresa, Brasil; MCZH, Museum of Comparative Zoology, Cambridge, EUA; MNRJ, Museu Nacional do Rio de Janeiro, Rio de Janeiro, Brasil; MZSP, Museu de Zoologia da Universidade de São Paulo, São Paulo, Brasil; SEMC, Snow Entomological Museum, Kansas, EUA; UFES, Coleção Entomológica do Departamento de Ciências Biológicas da Universidade Federal do Espírito Santo, Vitória, Brasil.

As distribuições geográficas das espécies estudadas e o número de espécies que ocorrem na região Neotropical foram obtidos, principalmente, a partir de Delvare (1992), De Santis (1979, 1980, 1983, 1989), De Santis \& Fidalgo (1994), Noyes (2002) e Arias \& Delvare (2003). Dados publicados posteriormente são citados oportunamente. A definição das regiões biogeográficas aqui utilizadas segue Morrone (2002).

A classificação supragenérica adotada segue Wijesekara (1997).

\section{Resultados}

Foram obtidas 149 espécies de Chalcididae pertencentes a 11 gêneros (Tabela 1). Desse total, 37 espécies foram relacionadas a nomes de espécies já descritas, que são listadas abaixo, juntamente com suas distribuições geográficas e novas ocorrências (localidades marcadas com asterisco, *). Ao menos 48 espécies encontradas não são descritas (espécies novas) (Tabela 1) e parte dessas já está sendo descrita em trabalhos específicos sobre os gêneros a que pertencem (M.T. Tavares, comunicação pessoal).

\section{Discussão}

Até o momento, apenas duas espécies, Notaspidium acutum e $N$. burdicki, tinham ocorrência conhecida para o Espírito Santo. Nove espécies são registradas aqui pela primeira vez para o Brasil. Arias \& Delvare (2003) compilaram 200 espécies de Chalcididae ocorrentes no Brasil. Marchiori et al. (2003) registraram quatro outras ocorrências e Tavares et al. (2006) descreveram duas espécies, totalizando 206 espécies para o país. Assim, o número de espécies nominais válidas de Chalcididae que ocorrem no Brasil passa a ser 215. O número de espécies nominais registradas para o Espírito Santo corresponde a $17 \%$ deste total, é superior as 28 espécies registradas para o Estado de São Paulo (Perioto \& Tavares 1999) e corresponde a $42 \%$ das 89 de espécies nominais registradas para a Colômbia (Arias \& Delvare 2003).

Com relação aos gêneros (Tabela 1), Halsteadium é registrado pela primeira vez para o Brasil, que passa a ter 18 gêneros, $61 \%$ deles com ocorrência no Espírito Santo. Aspirrhina, Conura, Epitranus, Stypiura e Zavoya também são novos registros para o Espírito Santo.

O total de 149 espécies obtidas é consideravelmente elevado, muito superior as 96 espécies registradas para a Colômbia (Arias \& Delvare 2003), as 96 registradas para a região Neártica e as 135 para a Europa (Noyes 2002). Nesses dois últimos casos é importante considerar que, apesar de se tratarem de espécies descritas, a fauna dessas regiões foi bem mais estudada e esses números devem corresponder a valores mais aproximados do número total de espécies existentes. 
Tabela 1. Número de gêneros e espécies de Chalcididae registrados para o Estado do Espírito Santo, Brasil.

Table 1. Number of genera and species of Chalcididae recorded to State of Espírito Santo, Brasil.

\begin{tabular}{|c|c|c|c|c|}
\hline Gênero & Total & $\begin{array}{c}\text { Espécies } \\
\text { identificadas }\end{array}$ & $\begin{array}{l}\text { Espécies novas } \\
\text { (mínimo) }\end{array}$ & $\begin{array}{l}\text { Espécies válidas } \\
\text { Neotropical }\end{array}$ \\
\hline Aspirrhina & 2 & 2 & 0 & 7 \\
\hline Brachymeria & 24 & 16 & 8 & 51 \\
\hline Conura & 89 & 6 & 19 & 279 \\
\hline Dirhinus & 1 & 1 & 0 & 13 \\
\hline Epitranus & 1 & 1 & 0 & 2 \\
\hline Halsteadium & 1 & 1 & 0 & 2 \\
\hline Haltichella & 6 & 2 & 4 & 6 \\
\hline Melanosmicra & 6 & 3 & 3 & 5 \\
\hline Notaspidium & 10 & 3 & 7 & 13 \\
\hline Stypiura & 7 & 1 & 6 & 6 \\
\hline Zavoya & 2 & 1 & 1 & 3 \\
\hline Total & 149 & 37 & 48 & 387 \\
\hline
\end{tabular}

Os três gêneros que apresentaram maior número de espécies no Espírito Santo (Conura, Brachymeria e Notaspidium) (Tabela 1) são aqueles considerados mais especiosos no Novo Mundo (Bouček 1992, Delvare 1992). O gênero Conura é extremamente especioso e, apesar da valiosa contribuição de Delvare (1992), a maior parte das espécies descritas ainda não é passível de identificação por falta de descrições adequadas. Esse fato impossibilitou interpretar se 64 das 89 espécies obtidas são descritas ou não.

Do total de espécies obtidas pelo menos um terço não está descrito. Para Notaspidium e Haltichella o número de espécies a serem descritas é superior a metade das espécies válidas para a região Neotropical. Para Stypiura, o número de espécies a serem descritas é igual ao de espécies válidas para a Neotropical. Esses valores mostram que o conhecimento da fauna de Chalcididae para o estado e para a região Neotropical está longe de ser satisfatório.

Com exceção de Conura, as espécies válidas registradas para cada gênero, no Espírito Santo, correspondem à cerca de um terço, ao menos, das espécies da região Neotropical, sendo proporcionalmente bem representativas.

A fauna do Espírito Santo é composta, em sua maior parte (26 das 37 espécies nominais), por espécies tipicamente neotropicais, e destas, nove têm ocorrência restrita ao Brasil (vide lista abaixo). Outras cinco espécies são predominantemente neotropicais, mas se estendem ao sul da região Neártica. Uma é tipicamente neártica, Brachymeria aeca, sendo aqui constatada pela primeira vez fora desta região. Epitranus clavatus aparenta ser originária dos trópicos do Velho Mundo, tendo sido introduzida no Novo Mundo repetidamente (Bouček 1982). Dirrhinus anthracia apresenta distribuição semelhante à espécie anterior e é constatada pela primeira vez para o Novo Mundo. Brachymeria podagrica é a espécie com distribuição mais ampla e já foi registrada em grande parte das regiões temperadas e tropicais do mundo. Brachymeria cabira era registrada apenas para as ilhas Galápagos e aqui é registrada pela primeira vez para o neotrópico continental. Até o momento, não foram constatadas espécies de Chalcididae endêmicas do Espírito Santo.

Apesar de preliminares, os dados aqui apresentados mostram que a fauna de Chalcididae do Espírito Santo é bastante rica e diversificada quando comparada com outras áreas já estudadas e corrobora com a riqueza já descrita para outros grupos de animais e plantas na região.

\section{Agradecimentos}

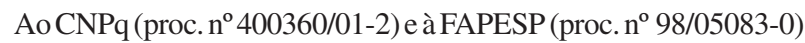
pelo apoio financeiro; ao FACITEC pela bolsa de iniciação científica concedida ao segundo autor. À Andrea Barbieri Navarro-Tavares pelo auxílio na identificação de parte das espécies de Melanosmicra. À Geane Oliveira Lannes pelo auxílio com a obtenção das coordenadas das localidades. Ao Ricardo Kawada pela doação de muitos dos exemplares estudados. Ao Dr. Celso O. Azevedo e ao revisor anônimo por sugestões e comentários. A Clarindo Krüger, José Carlos Lustoza, Juliberto Stur, Laerte Damasceno, Paulo Seick e Roberto Kautzky pela autorização de coleta em áreas de suas propriedades ou de sua responsabilidade. Aos curadores de coleções: E.C. Bergmann (IBSP), Carlos R. F. Brandão (MZSP), R.W.Brooks (SEMC), B. Dias (IBGE), M.S.Kelley (MCZH), C. Magalhães (INPA), W. Pulawsky (CASC).

\section{Referências Bibliográficas}

ARIAS, D.C. \& DELVARE, G. 2003. Lista de los géneros y especies de la família Chalcididae (Hymenoptera: Chalcidoidea) de la región Neotropical. Biota Colomb. 4(2):123-145.

AZEVEDO, C.O., KAWADA, R., TAVARES, M.T. \& PERIOTO, N.W. 2002. Perfil da Fauna de himenópteros parasitóides (Insecta, Hymenoptera) em uma área de Mata Atlântica do Parque Estadual da Fonte Grande, Vitória, ES, Brasil. Rev. Bras. Ent. 46(2):133-137.

AZEVEDO, C.O. \& SANTOS, E.S. 2000. Perfil da fauna de himenópteros parasitoides (Insecta, Hymenoptera) em uma área de Mata Atlântica da Reserva Biológica de Duas Bocas, Cariacica, ES, Brasil. Bol. Mus. Biol. Mello Leitão 11-12:117-126.

BOUČEK, Z. 1982. Oriental chalcid wasps of the genus Epitranus. J. Nat. Hist., 16:577-622.

BOUČEK, Z. 1988. Australasian Chalcidoidea (Hymenoptera): a biosystematic revision of the genera of fourteen families, with a reclassification of species. Wallingford: CAB International. 832p.

BOUČEK, Z. 1992. The New World genera of Chalcididae. Mem. Am. Entomol. Inst. 53:49-118.

BROWN JR., K.S. \& FREITAS, A.V.L. 2000. Diversidade de Lepidoptera em Santa Teresa, Espírito Santo. Bol. Mus. Biol. Mello Leitão 11-12: 71-116.

COWAN, D.P. 1979. The function of enlarged hind legs in oviposition and aggression by Chalcis canadensis (Hymenoptera: Chalcididae). The Great Lakes Entomol. 12:133-136. 
DE SANTIS, L. 1979. Catalogo de los Himenópteros Calcidoideos de América al sur de los Estados Unidos. Comisión de Investigacion Científica, La Plata.

DE SANTIS, L. 1980. Catalogo de los Himenópteros Brasileños de la serie parasitica: incluyendo Bethyloidea. Universidade Federal do Paraná, Curitiba.

DE SANTIS, L. 1983. Catalogo de los Himenopteros Calcidoideos de America al Sul de los Estados Unidos, Primer suplemento. Ver. Peruana Ent. 24(1):1-38.

DE SANTIS, L. 1989. Catalogo de los Himenopteroa Calcidoideos de America al Sul de los Estados Unidos, Segundo suplemento. Acta Entomol. Chil. 15:9-90.

DE SANTIS, L. \& FIDALGO, P. 1994. Catálogo de Himenopteros Calcidoideos. Academia Nacional de Agronomia y Veterinária, Buenos Aires.

DELVARE, G. 1992. A reclassification of the Chalcidini with a check list of the New World species. Mem. Am. Entomol. Inst. 53:119-442.

GIL-SANTANA, H.R. \& TAVARES, M.T. 2005. Brachymeria pandora (Crawford) (Hymenoptera, Chalcididae): a new parasitoid of Historis odius (Fabricius) (Lepidoptera, Nymphalidae). Rev. Bras. Zool. 22 (4):1211-1212.

GIL-SANTANA, H.R. \& TAVARES, M.T. 2006. Chalcidoid parasitoids (Hymenoptera) of Actinote parapheles Jordan, 1913 (Lepidoptera: Nymphalidae). Biota Neotrop. 6(1): http://www.biotaneotropica.org. br/v6n1/pt/abstract? short-communication+bn01006012006

HALSTEAD, J.A. 1991. New species of Notaspidium Dalla Torre from the Neartic and Neotropic regions. Pan-Pacific Entomol. 67(4):229-242.

MARCHIORI, C.H., PENTEADO-DIAS, A.M. \& TAVARES M.T. 2003. Parasitoids of the family chalcididae collected in pastures and forests using yellow traps, in Itumbiara, Goiás, Brasil. Braz. J. Biol. 63(2):357-360.
MARCHIORI, C.H., PEREIRA, L.A. \& FILHO, O.M.S. 2002. Brachymeria podagrica (Fabricius) (Hymenoptera: Chalcididae) como parasitoide de Chrysomya albiceps (Wiedemann) (Diptera: Calliphoridae): Primeiro relato no Brasil. Arq. Bras. Med. Vet. Zootec. 54(5):555-557.

MENDES, S.L. \& PADOVAN, M.P. 2000. A Estação Biológica de Santa Lúcia, Santa Teresa, Espírito Santo. Bol. Mus. Biol. Mello Leitão 11-12:7-34.

MORRONE, J.J. 2002. Biogeographical regions under track and cladistic scrunty. J. Biogeogr. 29:149-152.

NOYES, J.S. 2002. Interactive catalogue of world Chalcidoidea. Taxapad, Vancouver. (Banco de Dados em Cd-rom)

PASSAMANI, M., MENDES, S.L. \& CHIARELLO, A.G. 2000. Nom-volant mammals of the Estação Biológica de Santa Lúcia and adjacent areas of Santa Teresa, Espírito Santo, Brasil. Bol. Mus. Biol. Mello Leitão 11-12:201-214.

PERIOTO, N.W. \& M.T.TAVARES. 1999. Chalcidoidea. In Biodiversidade do Estado de São Paulo: Síntese do conhecimento ao final do século XX. Invertebrados Terrestres. (Brandão, C. R. F. \& Cancello, E.M. eds). FAPESP, São Paulo, p.153-168.

TAVARES, M.T., NAVARO-TAVARES, A.B. \& ALMEIDA, G. de S.S. 2006 The species of Chalcididae (Hymenoptera) parasitoids of Parides ascanius (Cramer), an endangered Papilionidae (Lepidoptera) from restingas of Southeastern Brazil. Zootaxa 1197:55-63.

THOMAZ, L.D. \& MONTEIRO, R. 1997. Composição florística da Mata Atlântica de encosta da Estação Biológica de Santa Lúcia, município de Santa Teresa-ES. Bol. Mus. Biol. Mello Leitão 7:3-48.

WIJESEKARA, G.A. 1997. Phylogeny of Chalcididae (Insecta: Hymenoptera) and its congruence with contemporary hierarchical classification. Contr. Amer. Entom. Inst. 29(3):1-61. 


\section{Anexo 1.}

\section{Lista de Espécies de Chalcididae do Espírito Santo}

* As localidades marcadas por asteriscos indicam novos registros de ocorrência

\section{Brachymerinae}

Brachymeria (Brachymeria) aeca Burks, 1960

Distribuição: Neártica e Neotropical* (Brasil: Espírito Santo).

Material examinado: BRASIL. Espírito Santo: 1 fêmea, Domingos Martins, P[arque]. Est[adual]. Pedra Azul, 20 25'55" S e $41^{\circ} 00$ ' 53" W, 26.XI-03.XII.2004, C. Azevedo e equipe col. (UFES).

Brachymeria (Brachymeria) annulata (Fabricius, 1793)

Distribuição: Amplamente distribuídos na região Neotropical. No Brasil é registrada nos estados: São Paulo, Goiás (Marchiori et al. 2003) e Espírito Santo*.

Material examinado: BRASIL. Espírito Santo: 08 fêmeas, 1 macho, Vitória, área urbana, $20^{\circ} 17^{\prime} 27^{\prime}$ ' S e $40^{\circ} 17^{\prime} 30^{\prime \prime} \mathrm{W}$, I.2001 (1), 28-30.VI.2003 (2), 22.I.2004 (1), 07-26.III.2004 (1), 1014.IV.2004 (3), 03-05.XII.2004 (1), R. Kawada col. (UFES); 1 fêmea,

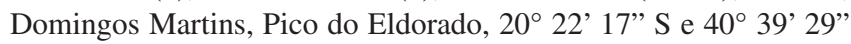
W, 03-10.XII.2004, M. Tavares e equipe col. (UFES).

\section{Brachymeria (Brachymeria) cabira (Walker, 1838)}

Comentários: Esta espécie foi descrita para o Arquipélago de Galápagos, onde ainda tem sido bastante amostrada. Alguns exemplares procedentes de outra áreas da região Neotropical tem sido estudados, inclusive do Espírito Santo.

Distribuição: Equador (Galápagos), Brasil* (Roraima, Distrito Federal, Espírito Santo, São Paulo), Bolívia e Argentina.

Material examinado: EQUADOR. Galápagos: 2 machos, Ilha Fernandina, Lado Oeste, 00 30' S e 90³0' W ii.1964, D.Q. Cavagnaro col. (CASC); 18 fêmeas, 4 machos, Ilha Santa Cruz, Estação de Pesq. Darwin, 00³0' S e 90³0' W, ii.1964 D.Q.Cavagnaro \& R.O.Schuster col. (CASC); 1 fêmea, idem, campo, D.Q. Cavagnaro col. (CASC); 4 fêmeas, Idem, Monte Mesa, 00³0' S e 90³0' W, 16.iv.1964, D.Q. Cavagnaro col. (CASC). BRASIL. Roraima: 1 fêmea, Rio Uraricoaera, Ilha de Maracá, 02-13.V.1987, Rafael e equipe col. (INPA). Distrito Federal: 1 fêmea, Brasília, Reserva Ecológica IBGE [=Roncador] 11-18.II.1982 (IBGE). Espírito Santo: 03 fêmeas, Vitória, área urbana, $20^{\circ} 17^{\prime} 27^{\prime}$ ' S e $40^{\circ} 17^{\prime} 30^{\prime \prime} \mathrm{W}$, 16.VI.2000, 23.VII.2001, 06.III.2002, R. Kawada col. (UFES). São Paulo: 1 fêmea, São Carlos, 1.IV.1956, C.D.Michener col. (SEMC); 1 fêmea, Pariquera-açú, Bandeja Branca [= Moericke], 31.V.1986, Expedição Inst[tituto] Biol[ógico] (IBSP). BOLÍVIA. Santa Cruz: 1 fêmea, Sara, Steinbach col. (MCZH). ARGENTINA. Salta: 1 fêmea, Cafayate, 5-6.I.1956, L.E. Peña col. (SEMC).

Brachymeria (Brachymeria) compacta (Walker, 1862)

Distribuição: México e Brasil* (Espírito Santo).

Material examinado: BRASIL. Espírito Santo: 02 fêmeas, Vitória, área urbana, 20 17’ 27' S e 40 17' 30”'W, 30.III-02.VI.2001 e 21.IV.2004, R. Kawada col. (UFES).

Brachymeria (Brachymeria) concitator (Walker, 1862)

Comentários: Arias \& Delvare (2003) citaram esta espécie apenas para o México, aparentemente com base na descrição original, porém a localidade-tipo é Brasil (Pará: Santarém).

Distribuição: México (Arias \& Delvare 2003) e Brasil (Pará, Espírito Santo*).
Material examinado: BRASIL. Espírito Santo: 1 fêmea, Pancas, Faz[enda]. Juliberto Stur, $19^{\circ} 12^{\prime} 54^{\prime \prime}$ S $40^{\circ} 47^{\prime}$ 52” W, arm. Malaise B6, 24-31.I.2003, M. T. Tavares e equipe col. (UFES); 1 fêmea, Cariacica, Res[erva] Biol[ógica]. Duas Bocas, $20^{\circ} 16^{\prime} \mathrm{S}$ e $40^{\circ} 28^{\prime} \mathrm{W}$, varredura de vegetação, 24.IX.1996, C. Azevedo e H. Santos col. (UFES); 01 macho, Domingos Martins, Pico do Eldorado, 20²2' 17' S e 40 39' 29' W, 03-10.XII.2004, M. Tavares e equipe col (UFES); 1 fêmea, A[tylio]. Vivacqua, Faz[enda]. J. C. Lustosa, $20^{\circ} 55^{\prime}$ 57' S e 41 ${ }^{\circ} 11^{\prime} 22^{\prime}$ ' W, arm. Malaise B3, 20-27. II.2003, M.Tavares, C. Azevedo e equipe col. (UFES).

Brachymeria (Brachymeria) flaviscapus (Girault, 1911)

Distribuição: Brasil* (Espírito Santo) e Paraguai.

Material examinado: BRASIL. Espírito Santo: 1 fêmea, Domingos Martins, Pico do Eldorado, 20²2' 17' S e 40 39' 29' W, 03-10.XII.2004, M. Tavares e equipe col (UFES).

Brachymeria (Brachymeria) koehleri Blanchard, 1935

Distribuição: Venezuela, Brasil e Argentina. No Brasil ocorre nos estados do Espírito Santo* e Rio de Janeiro (Tavares et al. 2006).

Material examinado: BRASIL. Espírito Santo: 01 fêmea, Vitória, área urbana, 20 17 ' 27” S $40^{\circ}$ e 17 ' 30” W, 15.XII.2004, R. Kawada col. (UFES).

Brachymeria (Brachymeria) mnestor (Walker, 1841)

Distribuição: Neártica e Neotropical. No Brasil ela é registrada para os estados do Amazonas, Espírito Santo*, Rio de Janeiro (Gil-Santana \& Tavares 2006, Tavares et al. 2006) e São Paulo.

Material examinado: BRASIL. Espírito Santo: 3 fêmeas, 4 machos, Vitória, área urbana, $20^{\circ} 17^{\prime} 27^{\prime}$ ' S e $40^{\circ} 17^{\prime} 30^{\prime \prime} \mathrm{W}$, 07-26.III.2004 (2), 29.IV.2004 (1), 01.VII.2004 (1), XI.2004 (2), [sem data] (1), R. Kawada col. (UFES); 3 fêmeas, Vitória, P[arque]. E[stadual]. da Fonte Grande, 20 18' S e 40 20' W, 29.I.2001 (1), 22.II.2001 (2), C. Azevedo e R. Kawada col. (UFES); 3 fêmeas, Cariacica, Re[serva]. Bio[lógica] Duas Bocas, 20 16' S e 40²8' W, 26.VIII.1996 (1), 24.IX.1996 (1), 06.V.2000 (1), C. Azevedo e H. Santos col. (UFES); 1 macho, idem, 24.VII.1999 (1), W.P. Souza col.(UFES); 02 fêmeas, Domingos Martins, Pico do Eldorado, $20^{\circ} 22^{\prime} 17$ ' S e 40 39' 29” W, 26.XI-03.XII.2004, M. Tavares e equipe col (UFES); 1 fêmea, Castelo, P[arque]. Est[adual]. Forno Grande, $20^{\circ} 31^{\prime} \mathrm{S}$ e $41^{\circ} 02$ ' W, 13.X.2000, H. Santos col. (UFES); 1 fêmea, Guarapari, Enseada Azul, 2040’S 40³0’W, [sem data], J. Gasparini col. (UFES).

Brachymeria (Brachymeria) nigritibialis Tavares \& Navarro-Tavares, 2006

Distribuição: Brasil (Espírito Santo* e Rio de Janeiro).

Material examinado: BRASIL. Espírito Santo: 7 fêmeas, 1 macho, Domingos Martins, Pico do Eldorado, 20 22' $17^{\prime}$ ' S e $40^{\circ} 39^{\prime}$ '29' W, 03-10.XII.2004, M. Tavares e equipe col (UFES).

Brachymeria (Brachymeria) pandora (Crawford, 1914)

Distribuição: Venezuela, Guiana e Brasil [Goiás (Marchiori et al. 2003), Espírito Santo*, Rio de Janeiro (Gil-Santana \& Tavares 2005)]

Material examinado: BRASIL. Espírito Santo: 3 fêmeas, 6 machos, Vitória, área urbana, $20^{\circ} 17^{\prime} 27^{\prime}$ ' S e $40^{\circ} 17^{\prime} 30^{\prime \prime} \mathrm{W}$, 12.III.2004 (1), 07-26.III.2004 (3), 14 e 29.IV.2004 (2), 3-11.XI.2004 (2), 27.XI.2004 (1), R. Kawada col. (UFES); 02 fêmeas, Domingos Martins, Pico do Eldorado, 20²2' 17' S e 40 39' 29”' W, 26.XI-10. XII.2004, M. Tavares e equipe col (UFES).

Brachymeria (Brachymeria) parvula (Walker, 1834) 
Distribuição: Estados Unidos, México, Colômbia (Arias \& Delvare 2003) e Brasil* (Espírito Santo).

Material examinado: BRASIL. Espírito Santo: 3 fêmeas, Vitória, área urbana, $20^{\circ} 17^{\prime} 27^{\prime}$ ' S e $40^{\circ} 17^{\prime} 30^{\prime \prime} \mathrm{W}, 28-30 . \mathrm{VI} .2003$, 22.I.2004, III.2004, R. Kawada col. (UFES).

Brachymeria (Brachymeria) podagrica (Fabricius, 1787)

Distribuição: Esta espécie parece estar distribuída por todas regiões tropicais e temperadas do mundo. Na região Neotropical ocorre no México, Cuba, Jamaica, Haiti, Venezuela e Brasil [Goiás, Minas Gerais (Marchiori et al. 2002), Espírito Santo*].

Material examinado: BRASIL. Espírito Santo: 09 fêmeas, Vitória, área urbana, $20^{\circ} 17^{\prime} 27$ ' S e $40^{\circ} 17^{\prime} 30^{\prime \prime} \mathrm{W}, 2001$ (1), 17.VI.2001 (1), 20.XI.2001 (1), 06.III.2002 (1), III.2004 (1), 3-5. XII.2004 (2), 17.I.2005 (2), R. Kawada col. (UFES). 1919)

Brachymeria (Pseudobrachymeria) annulipes (Costa Lima,

Distribuição: Brasil (Maranhão, Espírito Santo*)

Material examinado: BRASIL. Espírito Santo: 2 fêmeas, 1 ma-

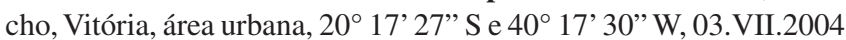
(1), 20.X-5.XII.2004 (2) R. Kawada col. (UFES).

Brachymeria (Pseudobrachymeria) pedalis (Cresson, 1872)

Distribuição: Estados Unidos, México e Brasil* (Espírito Santo).

Material examinado: BRASIL. Espírito Santo: 11 fêmeas, 1 macho, Vitória, área urbana, $20^{\circ} 17^{\prime} 27^{\prime \prime} \mathrm{S}$ e $40^{\circ} 17^{\prime} 30^{\prime \prime} \mathrm{W}$, 14.VI-20.VII.2001 (1), 20.X-20.XI.2001 (2), 10-29.VIII.2003 (1), 20.I.2004 (4), IX.2004 (1), 10-15.X.2001 (1), 04.XI.2004 (1), 20.XII.2004 (1), R. Kawada col. (UFES).

Brachymeria (Pseudobrachymeria) subconica Bouček, 1992

Distribuição: Regiões Neártica e Neotropical. No Brasil ocorre nos estados do Pará, Minas Gerais e Espírito Santo*.

Material examinado: BRASIL. Espírito Santo: 2 fêmeas,

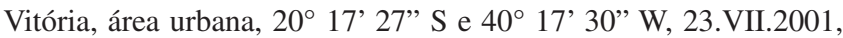
21.IV.2004, R. Kawada col. (UFES).

Brachymeria (Pseudobrachymeria) vesparum Bouček, 1992

Distribuição: Brasil (Pará, Espírito Santo*) e Argentina.

Material examinado: BRASIL. Espírito Santo: 3 fêmeas, Vitória, área urbana, $20^{\circ} 17$ ' 27" S e $40^{\circ} 17$ '30" W, 03.VII.2001, 26.III.2004, 3-5.XII.2004, R. Kawada col. (UFES).

Stypiura condalus (Walker, 1841)

Distribuição: Brasil (Pará, Espírito Santo*).

Material examinado: BRASIL. Espírito Santo: 1 fêmea, Cariacica, $\mathrm{Re}$ [serva]. Bio[lógica] Duas Bocas, $20^{\circ} 16^{\prime} \mathrm{S}$ e $40^{\circ} 28^{\prime} \mathrm{W}$, 23-25.XI.2001, R.Kawada col. (UFES); 1 fêmea, Corrego Itá, XI.1956, W. Zikás [=Zikán] col. (MNRJ).

\section{Chalcidinae}

Conura (Ceratosmicra) dema (Burks, 1940)

Distribuição: México e Brasil* (Espírito Santo).

Material examinado: BRASIL. Espírito Santo: 2 fêmeas, Linhares, Pontal do Ipiranga, $19^{\circ} 12^{\prime}$ S e $39^{\circ} 12^{\prime}$ W, 08-10.XII.2000, C. Azevedo e G. Schiffler col. (UFES).

Conura (Conura) maculata (Fabricius, 1787)

Distribuição: Neártica e Neotropical. No Brasil é registrada para os estados do Amazonas, Espírito Santo*, Rio de Janeiro e São Paulo.
Material examinado: BRASIL. Espírito Santo: 5 fêmeas, Vitória, área urbana, 20 17 ' 27" S e 40 17 ' 30” W, 2001 (1), 30.III-02.IV.2001 (1), 10-29.IV.2001 (1), 10-15.I.2005 (2), R. Kawada col. (UFES).

Conura (Conura) nigrifrons (Cameron, 1884)

Distribuição: Neártica e Neotropical. No Brasil é registrada para os estados do espírito Santo* e São Paulo.

Material examinado: BRASIL. Espírito Santo: 8 fêmeas, 1 macho, Vitória, área urbana, $20^{\circ} 17$ ' 27' S e $40^{\circ} 17$ ' 30" W, 20.X-20. XI.2001 (1), 28-30.VI.2003 (4), 20.XI.2004 (1), 22.I.2004 (3), R. Kawada col. (UFES). 19 fêmeas, 2 machos, Baixo Guandu, $19^{\circ} 30^{\prime} \mathrm{S}$ e $41^{\circ} 00^{\prime} \mathrm{W}$, VII.[19]71, P.Elias col. (MZSP).

Conura (Spilochalcis) femorata (Fabricius, 1775)

Comentários: Exemplares examinados foram criados de Autophila sp. (Noctuidae).

Distribuição: Neártica e Neotropical. No Brasil é registrada para os estados do Amazonas, Pernambuco, Espírito Santo* e São Paulo.

Material examinado: BRASIL. Espírito Santo: 6 fêmeas, 7 machos, Baixo Guandu, 19³0' S e 41 ${ }^{\circ} 00^{\prime}$ W, VII.[19]71, P.Elias col. (MZSP); 3 fêmeas, 3 machos, Porto Cachoeira [=Santa Leopoldina], $20^{\circ} 06^{\prime} \mathrm{S}$ e $40^{\circ} 31^{\prime} \mathrm{W}, \mathrm{IV} .1912$, em Authophila [= Autoplila, Noctuidae], E. Garbe col. (MZSP); 6 fêmeas, Vitória, área urbana, $20^{\circ} 17$ ' 27" S e $40^{\circ} 17$ ' 30" W, 7-26.III.2004 (2), 11.IV.2004 (1), 08.VIII.2004 (1), 10-11.XII.2004 (1), 17.I.2005 (1), R. Kawada col. (UFES); 1 fêmea, Cariacica, Re[serva]. Bio[lógica] Duas Bocas, $20^{\circ} 16^{\prime} \mathrm{S}$ e $40^{\circ} 28^{\prime}$ W, 05.XII.[19]96, C. Azevedo e H. Santos col. (UFES).

Conura (Spilochalcis) masus (Walker, 1841)

Comentários: O exemplar examinado é o primeiro registro de hospedeiro para a espécie e foi criado de Mechanitis lysimnia (Ithomiinae, Nymphalidae).

Distribuição: De Cuba e Guatemala até Peru, Bolívia e Brasil, onde é registrada para os estados do Amazonas, Espírito Santo* e São Paulo.

Material examinado: BRASIL. Espírito Santo: 1 macho, Vitória, Centro de Estudo Ambinetais, Companhia Siderúrgica de Tubarão, emergido de Mechanitis lysimnia (F.) [Ithomiinae, Nymphalidae] em Solanaceae, $20^{\circ} 17^{\prime}$ S e $40^{\circ} 17^{\prime}$ W, 11.I.2005, N. Marchesi col. (UFES).

Conura (Spilochalcis) minuta Delvare, 1992

Distribuição: Brasil (Paraíba, Espírito Santo*).

Material examinado: BRASIL. Espírito Santo: 01 fêmea, Domingos Martins, Pico do Eldorado, 20²2' 17' S e 40³9' 29” W, 26.XI-10.XII.2004, M. Tavares e equipe col (UFES).

Melanosmicra flavicollis (Cameron, 1904)

Distribuição: Neártica e Neotropical. No Brasil só é registrado para o estado do Espírito Santo*.

Material examinado: Santo: BRASIL. Espírito Santo: 1 fêmea, Linhares, Fazenda Maria Bonita, $19^{\circ} 25^{\prime} 50^{\prime \prime} \mathrm{S}$ e 39॰ 29' 40" W, varredura de vegetação, 11-13.VIII.2000, C.O. Azevedo e G. Schiffler col. (UFES); 2 fêmeas, Cariacica, Reserva Biológica de Duas Bocas, 26.XII.1996, C. Azevedo \& H. Santos col. (UFES); 9 fêmeas, idem, 17.IX.1996 (1), 05.XII.1996 (3), 25.III.1997 (2), 01.V.1997 (1), 22.X.1997 (1), 24.XI.1997 (1), H. Santos col. (UFES).

Melanosmicra gracilis (Kirby, 1889)

Distribuição: Brasil (Espírito Santo*, Rio de Janeiro). 
Material examinado: BRASIL. Espírito Santo: 1 macho, Santa Teresa, Est[ação]. Bio[lógica] Santa Lúcia, 19 58' 18" S e 40³2' 07' W, arm. Malaise, 06-09.IV.2001, C. O. Azevedo e eq[uipe]. col. (MZSP); 1 macho, idem, varredura de vegetação, 28.III.2001, C. Azevedo e R. Kawada col. (UFES); 2 macho, Domingos Martins, Mata Pico do Eldorado, 20 22' $17^{\circ}$ ' S e $40^{\circ} 39^{\prime} 29^{\prime}$ 'W, arm. Malaise, 26.XI-03.XII.2004 e 03-10.XII.2004, M.T.Tavares e eq[uipe]. Col. (UFES).

Melanosmicra immaculata Ashmead, 1904

Distribuição: Brasil (Mato Grosso, Espírito Santo*).

Material examinado: BRASIL. Espírito Santo: 1 fêmea, Vitória, P[arque] Est[adual] Fonte Grande, varredura de vegetação, 26.III.2001, C. Azevedo e R. Kawada col. (UFES); 1 fêmea, Cariacica, Reserva Biológica de Duas Bocas, varredura vegetação, 25.II.1997, C. Azevedo e H. Santos col. (UFES); 1 fêmea, idem, 26.ix.2005, C. Azevedo e eq[uipe]. col. (UFES); 1 fêmea, Alegre, P[arque] Est[adual] Cachoeira da Fumaça, 12.X.2000, Santos Sá col. (UFES); 2 fêmeas, Domingos Martins, Mata Pico do Eldorado, 20²2'17' S e 40 39'29" W, arm. Malaise, 26.XI-03.XII.2004, M. T. Tavares e eq[uipe]. col. (UFES).

\section{Dirhininae}

Dirhinus anthracia Walker, 1846

Comentários: Da mesma forma que B. pandora, que ataca dípteros sinantrópicos, esta espécie está distribuída em muitas regiões do mundo e é possível que ocorra em outras localidades neotropicais.

Distribuição: Oriental, Afrotropical, Afrotemperada, Neotropical, Neoguineana, Australotropical e Australotemperada. Na região Neotropical é registrado apenas para o Brasil* (Espírito Santo).

Material examinado: BRASIL. Espírito Santo: 1 fêmea, Vitória, área urbana, 20 17' 27”'S e 40 17’30” W, 10-11.XII.2004, R. Kawada col. (UFES).

\section{Epitraninae}

Epitranus clavatus (Fabricius, 1804)

Comentários: Bouček (1992) discute a ocorrência desta espécie nas Américas e afirma que a mesma é associada a microlepidópteros, inclusive Àqueles se desenvolvendo em produtos estocados (p.e. grãos armazenados). Todos os exemplares procedentes do Brasil e analisados por nós advêm de localidades com atividades portuárias e parecem ser esporadicamente amostrados. Isso sugere que a espécie não deve ter se estabelecido no país e os exemplares coletados advém diretamente de hospedeiros de produtos importados.

Distribuição: Neártica, Oriental, Afrotropical e Neotropical. No Brasil é registrada para os estados de Espírito Santo*, Rio de Janeiro* e São Paulo*.

Material examinado: BRASIL. Espírito Santo: 1 fêmea, Vitória, área urbana, 20 17 ' 27' S e 40 17’30” W, 2001, R. Kawada col. (UFES). Rio de Janeiro: 2 fêmeas, Rio de Janeiro, III.1925, D. Mendes col. (MNRJ); 2 machos, Rio de Janeiro, Praia Vermelha, 22 ${ }^{\circ} 56^{\prime} \mathrm{S}$ e $43^{\circ} 09^{\prime} \mathrm{W}, 18$ e 20.X.1930 (MNRJ); 1 fêmea, Estrada Rio Santo km 47 [=Mangaratiba], $22^{\circ} 47^{\prime}$ S e 44 02' W, 24.IX.1948, J.Miranda col. (MNRJ).

\section{Haltichellinae}

Aspirrhina dubitator (Walker, 1862)

Distribuição: Brasil (Roraima, Amazonas, Pará, Mato Grosso, Espírito Santo).

Material examinado: BRASIL. Espírito Santo: 2 machos, Conceição da Barra, P[arque]. Est[adual]. Itaúnas, $18^{\circ} 20^{\prime} \mathrm{S}$ e 39 40’ W, 10-15.I.2005, R. Kawada col. (UFES).
Aspirrhina remotor (Walker, 1862)

Comentários: Bouček (1992) sugeriu que Aspirhina [=Aspirrhina] deceptor Halstead, 1991 pode ser um sinônimo de A. remotor e não propôs formalmente tal sinonímia. Assim, como ainda não foi confirmada, essa sinonímia não é considerada aqui.

Distribuição: Brasil (Pará, Espírito Santo*) e Argentina.

Material examinado: BRASIL. Espírito Santo: 1 fêmea, Domingos Martins, Pico do Eldorado, 20²2' $17^{\prime \prime}$ S e 40 39' 29'” W, 26.XI-03.XII.2004, M. Tavares e equipe col (UFES).

Halsteadium petiolatum Bouček, 1992

Distribuição: Guatemala, Costa rica, Colômbia, Trinidad e Tobago, e Brasil* (Espírito Santo).

Material examinado: BRASIL. Espírito Santo: 1 fêmea, Castelo, P[arque]. E[stadual]. Forno Grande, $20^{\circ} 31^{\prime} \mathrm{S}$ e $41^{\circ} 02^{\prime} \mathrm{W}$, 15.X.2000, C. Azevedo col. (UFES); 2 fêmeas, Santa Maria Jetibá, F[azenda] Clarindo Krüger, $20^{\circ} 04^{\prime} 27^{\prime}$ 'S e $40^{\circ} 44^{\prime} 51^{\prime}$ ' W, Malaise 06-13.XII.2002, MTavares, C.Azevedo e equipe col. (UFES).

Haltichella hydara (Walker, 1842)

Distribuição: Venezuela (Arias \& Delvare 2003) e Brasil (Espírito Santo*).

Material examinado: BRASIL. Espírito Santo: 1 fêmea, Santa Tersa, Museu Mello Leitão, $1^{\circ}$ 35' S e 40³6' W, 26.VII.1988, A.P. Aguiar col. (MBML); 1 macho, Castelo, P[arque]. Est[adual]. Forno Grande, $20^{\circ} 31^{\prime} \mathrm{S}$ e $41^{\circ} 02^{\prime} \mathrm{W}$, varredura, 13.X.2000, H. Santos col. (UFES); 1 macho, Alegre P[arque] Est[adual] C[achoeira] da Fumaça, varredura, $20^{\circ} 37^{\prime} 41^{\circ} 36^{\prime}$ W, 12.X.2000, H.S. Santos col. (UFES).

\section{Haltichella ornaticornis Cameron, 1884}

Distribuição: Neártica e Neotropical. No Brasil é registrada para os estados de Goiás (Marchiori et al. 2003) e Espírito Santo*.

Material examinado: BRASIL. Espírito Santo: 3 machos, Linhares [=Sooretama], Res[erva] Biol[ógica] Sooretama, $19^{\circ} 00^{\prime} 11^{\prime}$ ' S e 40 07' 08” W, varredura, 22.III.2002 (2), 06.VI.2002 (1), C.O. Azevedo col. (MZSP); 1 macho, Linhares, R.B. [= Floresta Nacional] Goytacazes, $19^{\circ} 26^{\prime} \mathrm{S}$ e $40^{\circ} 04^{\prime} \mathrm{W}$, rede [varredura], 06-08.X.2000 (UFES); 1 macho, Pancas, Faz[enda] Juliberto Stur, $19^{\circ} 13$ ' 10" S e 40 46' 23" W, Malaise, 24-31.I.[20]03, M Tavares e eq[uipe] col. (UFES); 2 machos, Linhares, Povoação, Fazenda Benesforte, restinga arbórea, varredura, 08-10.X.2000, C.Azevedo e G.Schiffer col. (UFES); 1 macho, Santa Teresa, Est[ação] Biol[ógica] Santa Lúcia, $19^{\circ} 59^{\prime} \mathrm{S}$ e $40^{\circ} 20^{\prime} \mathrm{W}$, varredura, 29.VI.2001, C. Azevedo e R. Kawada col. (UFES); 3 machos, Vitória, P[arque]. E[stadual]. Fonte Grande, $20^{\circ} 18^{\prime} \mathrm{S}$ e $40^{\circ} 20^{\prime} \mathrm{W}, 24 . X I .2000$ e 22.II.2001, C. Azevedo e R. Kawada col. (UFES); 6 fêmeas, 5 machos, Cariacica, $\mathrm{R}$ [eserva]. B [iológica] Duas Bocas, 20 16' S e 40 28' W, varredura, 02.VIII.1996 (1), 09.IX.1996 (3), 22.X.1996 (2), 12.XI.1996 (3), 29.I.1997 (1), 06.II.1997 (1), C. Azevedo e H. Santos col. (UFES); 1 fêmea, Alegre P[arque] Est[adual] C[achoeira] da Fumaça, varre-

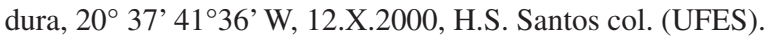

\section{Notaspidium acutum Halstead, 1991}

Distribuição: Colômbia, Brasil (Amazonas, Mato Grosso, Espírito Santo) e Equador.

Material examinado: BRASIL. Espírito Santo: 1 macho, Santa Teresa, Est[ação]. Bio[lógica]. Santa Lúcia, 19 58' 18" S e 40 32' 07' W, varredura, 29.XI-06.XII.2002, C. Azevedo e R. Kawada col. (UFES); 1 macho, Santa Maria de Jetibá, Faz[enda] Paulo Seick, 2002'31'S 4041'51'W, arm. Malaise, 29.XI-06.XII.2002, M. T.Tavares e eq[uipe]. col. (UFES); 1 macho, Santa Maria de Jetibá, Faz[enda] Clarindo Krüger, 20 04' 27 " S e $40^{\circ} 44^{\prime}$ 51" W, arm. Malaise, 29.XI-06.XII.2002, M. T. Tavares, C. 
O. Azevedo e eq[uipe]. col. (UFES); 2 macho, Cariacica, Re[serva] Bio[lógica] Duas Bocas, 20 16' S e 40²8' W, varredura, 04.X.1996 e 16.I.1997, Santos Sá col. (UFES); idem, 1 fêmea, 26.XII.1996, C. Azevedo e Santos col. (UFES).

Notaspidium burdicki Halstead, 1991

Distribuição: Brasil (Mato Grosso, Espírito Santo, Rio de Janeiro).

Material examinado: BRASIL. Espírito Santo: 1 macho, Santa Teresa, Est[ação]. Bio[lógica]. Santa Lúcia, 19 58' 25" S e 40³1' 44" W, varredura, 8.IV.2001, C. Azevedo e eq[uipe]. col. (MZSP); 1 macho, Vitória, P[arque]. Est[adual]. Fonte Grande, $20^{\circ} 18^{\prime} \mathrm{S}$ e $40^{\circ} 20^{\prime} \mathrm{W}$, varredura, 22.II.2001, C. Azevedo e R. Kawada col. (UFES); 1 macho, Domingos Martins, Pico do Eldorado, $20^{\circ} 22^{\prime} 17^{\prime}$ 'S e $40^{\circ} 39^{\prime}$ '29” W, arm. Malaise, 26.XI-03.XII.2004, M. T. Tavares e eq. col. (UFES).
Notaspidium giganteum Halstead, 1991

Distribuição: Neotropical. No Brasil é registrada para os estados: Espírito Santo* e São Paulo.

Material examinado: BRASIL. Espírito Santo: 1 macho, Vila Velha, Área Urbana, 04.IX.1989, A.P. Aguiar col. (MBML).

Zavoya cooperi Bouček, 1992

Distribuição: Costa Rica, Panamá, Colômbia, Venezuela (Arias \& Delvare 2003), Trinidad \& Tobago e Brasil (Bahia, Minas Gerais, Espírito Santo*).

Material examinado: BRASIL. Espírito Santo: 1 fêmea, Santa Teresa, Est[ação] Biol[ógica] Santa Lúcia, 1959' S e 40²0’ W, Moericke, 09-12.IV.2001, C. Azevedo e eq[uipe] col. (MZSP); idem, 1 fêmea, Moericke, 27-31.VI.2004, M.Tavares col (UFES); 1 fêmea, Vitória, P[arque]. E[stadual]. Fonte Grande, $20^{\circ} 18^{\prime} \mathrm{S}$ e $40^{\circ} 20^{\prime} \mathrm{W}$, varredura, 30.VI.2000 C. Azevedo e R. Kawada col. (UFES); idem, 1 fêmea, 04.I.2001, C.Azevedo, R. Kawada e H.Santos col. (UFES); 1 fêmea, Cariacica, R[eserva]. B[iológica] Duas Bocas, 20¹6’ S, e 40²8’W, varredura, 05.XII.1996, Santos Sá col. (UFES).

Título: Espécies de Chalcididae (Hymenoptera, Insecta) do Estado do Espírito Santo, Brasil.

Autores: Tavares MT \& Araujo BC

Biota Neotropica, Vol.7 (número 2): 2007

http://www.biotaneotropica.org.br/v7n2/pt/abstract?inventory +bn02207022007

Recebido em 01/09/06 - Versão reformulada recebida em 15/03/07 - Publicado em 04/06/07

ISSN 1676-0603 